\title{
The HIV-I Vpr and glucocorticoid receptor complex is a gain of function interaction that prevents the nuclear localization of PARP-I
}

\author{
Karuppiah Muthumani*1, Andrew Y Choo ${ }^{1}$, Wei-Xing Zong2, \\ Muniswamy Madesh², Daniel S Hwang ${ }^{1}$, Arumugam Premkumar ${ }^{3}$, \\ Craig B Thompson ${ }^{2}$ and David B Weiner ${ }^{1}$
}

Address: ${ }^{1}$ Department of Pathology and Laboratory Medicine, University of Pennsylvania School of Medicine, Philadelphia, Pennsylvania, 19104, USA, ${ }^{2}$ Abramson Family Cancer Research Institute, Department of Cancer Biology, University of Pennsylvania School of Medicine, Pennsylvania, 19104, USA and ${ }^{3}$ Laboratory of Molecular Neuropharmacology, Memorial Sloan-Kettering Cancer Center, New York, New York, 10021, USA

* Corresponding author

from 2006 International Meeting of The Institute of Human Virology

Baltimore, USA. 17-2I November, 2006

Published: 21 December 2006

Retrovirology 2006, 3(Suppl I):SI05 doi:10.1186/1742-4690-3-SI-SI05

(C) 2006 Muthumani et al; licensee BioMed Central Ltd.

The Vpr protein of HIV-1 functions as a vital accessory gene by regulating various cellular functions including

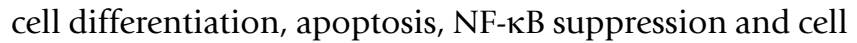
cycle arrest of the host cell. Several reports have suggested that Vpr complexes with the glucocorticoid receptor (GR), but it remains unclear whether the GR pathway is required for Vpr's effects. Here we report that Vpr utilizes the GR pathway as a recruitment vehicle for the NF- $\mathrm{kB}$ coactivating protein Poly(ADP-Ribose) Polymerase-1 (PARP-1). The glucocorticoid receptor interaction with $\mathrm{Vpr}$ is both necessary and sufficient to facilitate this interaction by potentiating the formation of a Vpr/GR/PARP-1 complex. The recruitment of PARP-1 by the Vpr/GR complex prevents its nuclear localization, which is necessary for Vpr to suppress NF-kB. The association of GR with PARP-1 is not observed with steroid (glucocorticoid) treatment, suggesting that the GR association with PARP-1 is a gain of function solely attributed to HIV-1 Vpr. These data provide important insight into Vpr biology and its role in HIV pathogenesis. 